\title{
PERTURBATIONS OF THE DEPTH OF LIQUID PENETRATION INTO THE CAPILLARY DURING BUBBLE DEPARTURES
}

\author{
Paweł DZIENIS ${ }^{*}$ \\ * Department of Mechanics and Applied Computer Science, Faculty of Mechanical Engineering, \\ Białystok University of Technology ul. Wiejska 45C, 15-351 Białystok, Poland \\ p.dzienis@pb.edu.pl
}

received 19 September 2021, revised 18 October 2021, accepted 22 October 2021

\begin{abstract}
In the present paper, the influence of bubble size on liquid penetration into the capillary was experimentally and numerically studied. In the experiment, bubbles were generated from a glass capillary (with an inner diameter equal to $1 \mathrm{~mm}$ ) in a glass tank containing distilled water, tap water or an aqueous solution of calcium carbonate. These liquids differ in the value of their surface tension, which influences the bubble size. During experimental investigations, air pressure fluctuations in the gas supply system were measured. Simultaneously, the videos showing the liquids' penetration into the capillary were recorded. Based on the videos, the time series of liquid movements inside the capillary were recovered. The numerical models were used to study the influence of bubble size on the velocity of liquid flow above the capillary and the depth of liquid penetration into the capillary. It was shown that the air volume flow rate and the surface tension have the greatest impact on the changes of pressure during a single cycle of bubble departure $(\Delta p)$. The changes in pressure during a single cycle of bubble departure determine the depth of liquid penetration into the capillary. Moreover, the values of $\Delta p$ and, consequently, the depth of liquid penetration can be modified by perturbations in the liquid velocity above the capillary outlet.
\end{abstract}

Keywords: bubbles, bubble departures, liquid movement into the capillary

\section{INTRODUCTION}

The process of gas flow in a liquid is a very complex problem. This process has been researched in many scientific fields: chemical and process engineering, pharmaceuticals, food production and fertiliser production. Understanding the phenomenon of gas flow in a liquid allows better control of processes associated with aeration or saturation (Cano-Lozano et al., 2017). Moreover, knowledge of the flow of gas bubbles in underwater environments is used in the estimation of the global balance of greenhouse gases (Leifer and Tang, 2007; Vázquez et al., 2015).

Research of gas bubbles in liquids concerns the following: bubble formation (Aoyama et al., 2016, Cano-Lozano et al., 2017), bubble coalescence (Farhat et al., 2021), bubble flow and its trajectories (Liu et al., 2015; Augustyniak and Perkowski, 2021) or chaotic bubble behaviour (Zang and Shoji, 2001; Cieslinski and Mosdorf, 2005; Mosdorf and Shoji, 2003). The time period of bubble formation may be divided into two periods: waiting time and time of bubble growth. During the waiting time (for the low air volume flow rates supplied to the capillary or orifice), the capillary is flooded by liquid, caused by a decrease in pressure in the gas supply system after the bubble departure stage (Ruzicka et al., 2009a; Dzienis and Mosdorf, 2014; Cano-Lozano et al., 2017). Subsequently, the liquid is removed from the capillary because the gas pressure inside the gas supply system increases (Koval'chuk et al., 1999).

In previous papers (Ruzicka et al., 2009a, 2009b; Stanovsky et al., 2011; Dzienis and Mosdorf, 2014; Cano-Lozano et al., 2017), the liquid movement inside the orifice or capillary, after bubble departure, was experimentally and numerically investigated. In other papers (Dukhin et al., 1998a; Dukhin et al., 1998b; Koval'chuk, 1999; Ruzicka et al., 2009a; Stanovsky et al., 2011), the influence of plate thickness, orifice diameter, gas chamber volume (volume of gas supply system), height of the liquid column above the orifice outlet, surface tension and viscosity of the liquid on liquid penetration into the orifice or capillary was investigated. In the paper by Ruzicka et al., (2009a), it was concluded that an increase in the chamber volume causes an increase in the time period between two subsequent bubbles. Moreover, an increase in height of the liquid column over the orifice outlet leads to an increase in time period between subsequent bubbles. Ruzicka et al., (2009a, 2009b) experimentally and numerically studied the oscillations of the gas-liquid interface inside the orifice and showed that the gas-liquid interface inside the orifice modifies the duration of bubble formation. This period decreases when the number of oscillations of the gas-liquid interface decreases. Dzienis and Mosdorf (2014) showed that an increase in the volume of the gas supply system causes an increase in the maximum depth of liquid penetration into the capillary. Moreover, chaotic bubble departures are caused by changes in the velocity of liquid flow above the capillary. It can be assumed that perturbations of liquid flow can be amplified by changes in the size of subsequent departed bubbles and consequently cause chaotic variations in the depth of liquid penetration into the capillary.

In the present paper, the influence of the size of a departed bubble on the liquid's velocity and depth of liquid penetration into the capillary was experimentally and numerically studied. In the experiment, bubbles were generated from a glass capillary in a glass tank containing distilled water, tap water or an aqueous 
solution of calcium carbonate. These liquids are distinguished by their values of surface tension, which influence the size of the departed bubbles. In the numerical simulation, the influence of bubble size on the liquid's velocity above the capillary outlet and on the depth of penetration of the liquid into the capillary was studied. It is shown that the air volume flow rate and the surface tension have the greatest impact on the pressure changes $(\Delta p)$ during a single cycle of bubble departure. These changes determine the depth of liquid penetration into the capillary. The values of $\Delta p$ and, consequently, the depth of liquid penetration can be modified by variations in the liquid's velocity above the capillary outlet.

In Section 2 of this paper, the experimental setup and data characteristics are described. Results of the experimental data analysis are shown in Section 3. In Section 4, numerical simulation and its results are described. Finally, in Section 5, the conclusion is given.

\section{EXPERIMENTAL SETUP AND DATA CHARACTERISTICS}

In the experimental investigations, bubbles were generated in a glass tank $(300 \times 150 \times 700 \mathrm{~mm})$. The schema of the experimental setup is shown in Fig. 1.

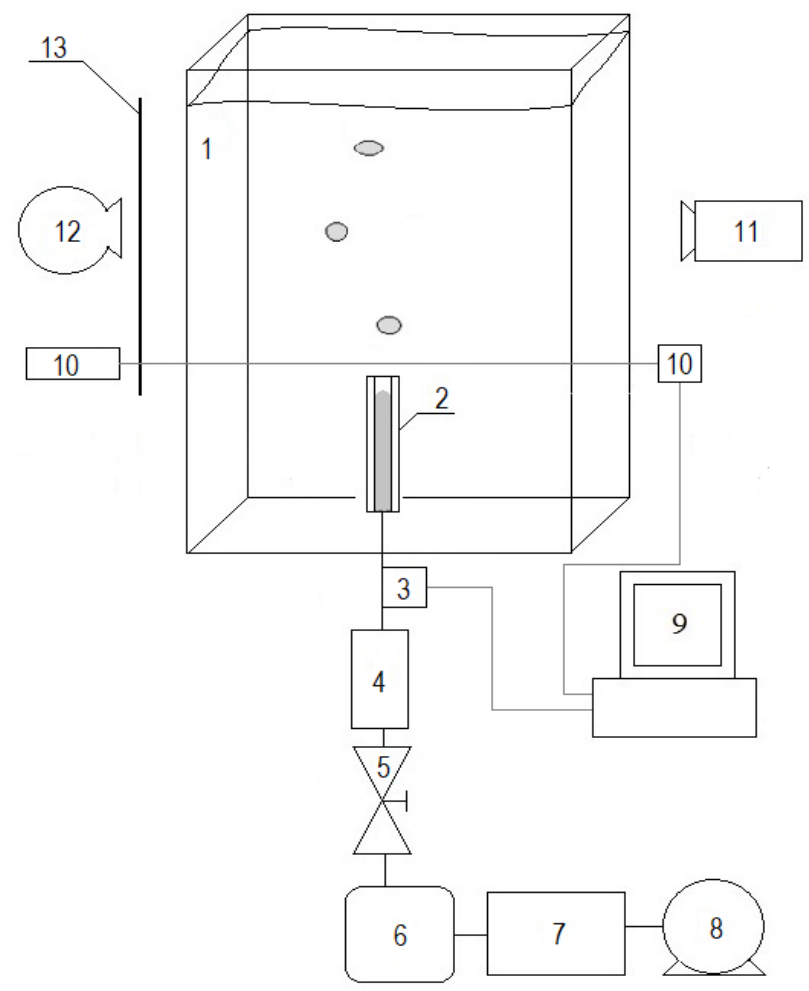

Fig. 1. Experimental setup: 1 - glass tank, 2 - glass capillary, 3 - pressure sensor, 4 - rotameter, 5 - air valve, 6 - air tank, 7 - pressure regulator, 8 - air pump, 9 - computer acquisition system, 10 - laser - phototransistor system, 11 - high speed camera, 12 - light source, 13 - screen.

Bubbles were generated from a glass capillary with inner diameter equal to $1 \mathrm{~mm}$ in a glass tank containing distilled water, tap water and aqueous calcium carbonate (c-c in aqueous solution). The surface tension was measured using an STA-1 tensiometer. The accuracy of the tensiometer is equal to $0.1 \mathrm{mN} / \mathrm{m}$. Surface tension of distilled water was $65.3 \mathrm{mN} / \mathrm{m}$, tap water was
$72.2 \mathrm{mN} / \mathrm{m}$ and calcium carbonate in aqueous solution was $75.4 \mathrm{mN} / \mathrm{m}$.

The capillary was placed at the bottom of the tank. In the first experiment, the tank was filled with distilled water; in the next experiment, the water was removed and the tank was filled with tap water. In the last experiment, the tank was filled with c-c aqueous solution. In all experiments, the liquid temperature was measured with a digital thermometer MAXIM DS18B20 (with an accuracy of $0.1^{\circ} \mathrm{C}$ ) and was equal to $20^{\circ} \mathrm{C}$. During the experimental investigations, the air volume flow rate was changed. The air volume flow rate was set in the following ranges: for distilled water, 0.005-0.026 I/min; for tap water, 0.005-0.038 I/min; and for $c-c$ aqueous solution, $0.005-0.042 \mathrm{l} / \mathrm{min}$. The step change in air volume flow rate was $0.003 \mathrm{l} / \mathrm{min}$. The air volume flow rates were selected such that liquid penetration into the capillary occurred. In the experiments, the air pressure fluctuations in the gas supply system and videos of the process of liquid penetration into the capillary were recorded. The air pressure fluctuations were measured using the silicon pressure sensor MPX12DP and recorded using the data acquisition system DT9800 series with a sampling frequency of $1 \mathrm{kHz}$. The pressure in the air tank was set by a proportional pressure reducing valve Metalwork Regtronic. During the experimental investigation, the air pressure was set as 0.3 bar, with an accuracy equal to $0.5 \%$.

The bubble departure process and liquid penetration into the capillary were recorded with a Phantom v1610 high-speed camera. The duration of each video was $30 \mathrm{~s}$. The videos were recorded in grey scale, with a speed of $5,000 \mathrm{fps}$ (frames per second). Videos were divided into frames. The example and selected frames of videos containing one cycle of bubble departure are shown in Fig. 2a. The depth of liquid penetration inside the capillary was measured using a computer programme. The programme counts, on each frame, the number of pixels with high brightness. Bright pixels represent the occurrence of water in the capillary. That programme allows one to receive the time series of liquid penetration into the capillary.

Data from both the high-speed camera and the acquisition station were synchronised using the laser-phototransistor system. The method of synchronisation was described in a previous paper (Dzienis and Mosdorf, 2013). In order to standardise the sampling frequency of the time series of liquid penetration into the capillary and the pressure fluctuations, the time series of pressure fluctuations were re-sampled using a computer programme. Examples of synchronised time series of liquid penetration into the capillary and the pressure fluctuations, for one cycle of bubble departure, are shown in Fig. $2 b$. The bubble departure occurs at the lowest value of pressure $\left(p_{\min }\right)$ in the air supply system. During the process of liquid penetration into the capillary, the pressure increases to the maximum value $\left(p_{\max }\right)$. The onset of bubble growth occurs at the maximum value of pressure $\left(p_{\max }\right)$, and it causes a decrease of pressure in the gas supply system. It can be concluded that the depth of liquid penetration into the capillary is dependent on pressure fluctuations during one cycle of bubble departure $(\Delta p)$ (Fig. 2).

When bubbles depart in tap water and c-c aqueous solutions, then increase of air volume flow rate causes the maximum depth of liquid penetration into the capillary to decrease (Fig. 3a). When bubbles depart in distilled water, then for changes in the air volume flow rates within the range of $0.008-0.026 \mathrm{l} / \mathrm{min}$, the maximum depths of liquid penetration were similar to each other. The value of maximum depth of liquid penetration fluctuated in the range of $6.2-6.7 \mathrm{~mm}$ (Fig. 3a). 
a)

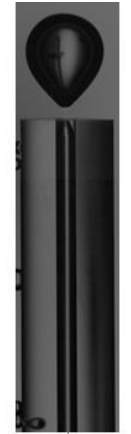

b)

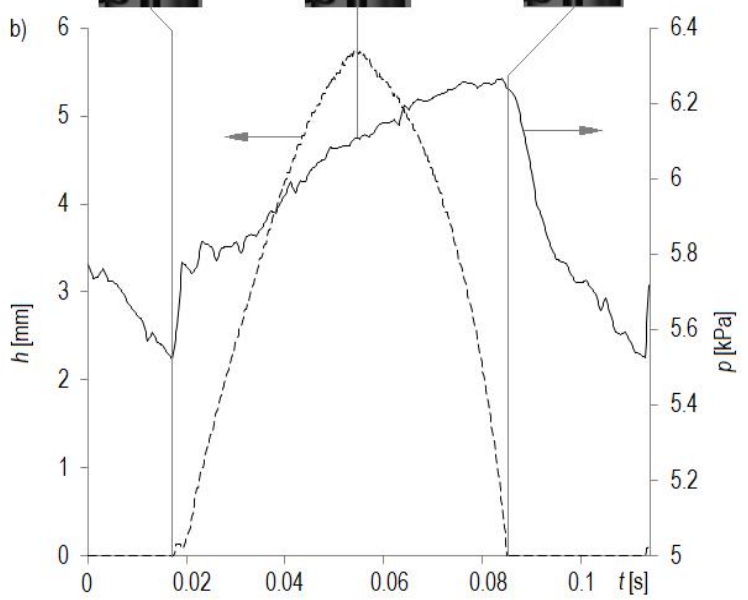

Fig. 2. Examples of video frames and synchronised time series of liquid penetration into the capillary and the pressure fluctuations, for one cycle of bubble departure: (a) frames of videos; (b) time series of liquid penetration into the capillary (dotted line) and the pressure fluctuations (continuous line)
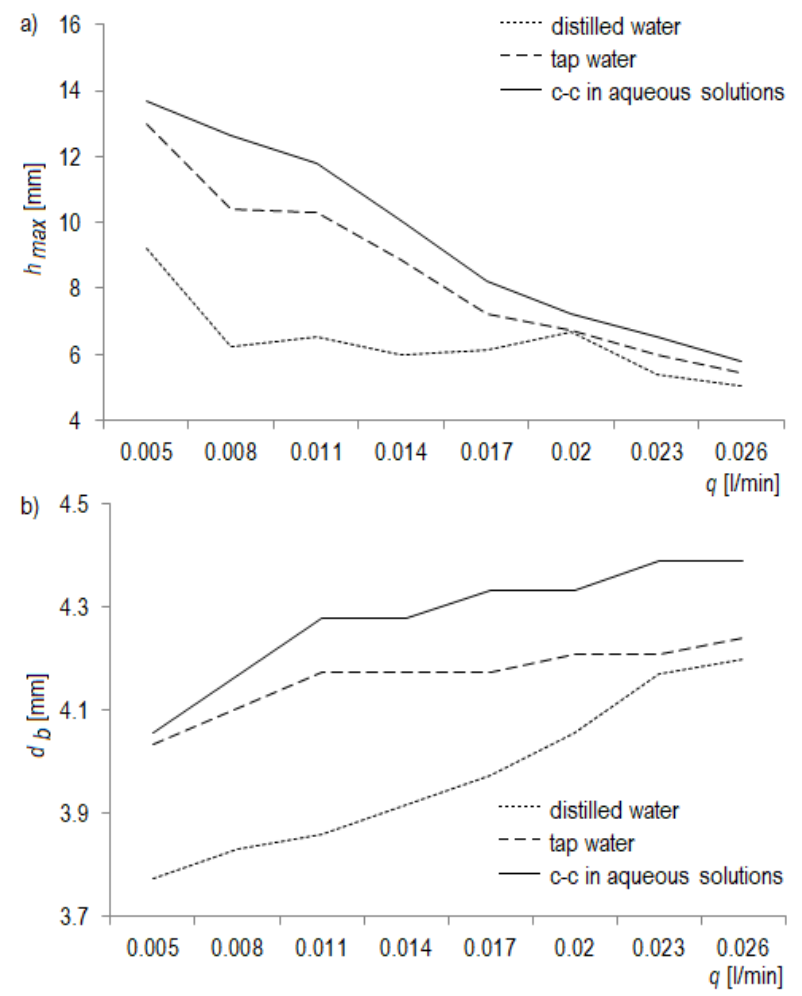

Fig. 3. The changes in maximum depth of liquid penetration into the capillary and departed bubble diameter vs. air volume flow rate:

(a) maximum depth of liquid penetration into the capillary;

(b) bubble diameter just after its departure.
Changes in the bubble diameter (just after its departure) vs. air volume flow rate are shown in Fig. $3 \mathrm{~b}$. Increase of air volume flow rate causes an increase in the diameters of departed bubbles. The bubble diameter increase vs. increase in air volume flow rate is different for the different types of water investigated. The largest increase in diameter of departed bubbles was obtained for bubbles generated in distilled water.

The changes of $\Delta p$ vs. air volume flow rate is presented in Fig. 4. The values of $\Delta p$ are greater for bubbles generated in tap water and $\mathrm{c}-\mathrm{c}$ aqueous solution than for bubbles generated in distilled water. Moreover, an increase in air volume flow rate causes a downward trend in the values of $\Delta p$ for the bubbles departed in tap water and $\mathrm{c}-\mathrm{c}$ aqueous solution. Another situation is observed in the case of bubble departure in distilled water. The increase of air volume flow rate causes a slight increase in $\Delta p$. It can be concluded that the shallower depth of distilled water in the capillary is caused by lower fluctuations of air pressure during a single cycle of bubble departures.

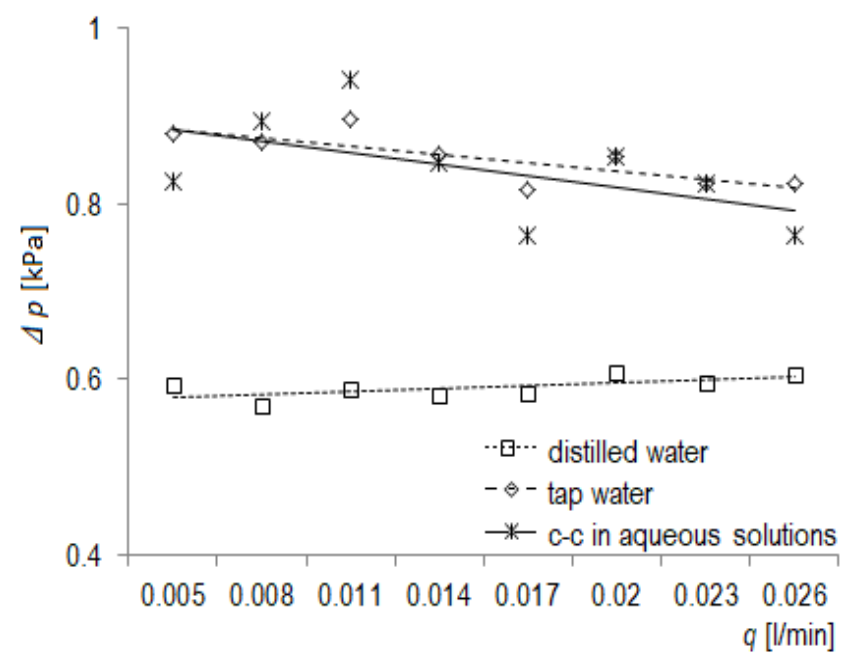

Fig. 4. The changes of $\Delta p$ vs. air volume flow rate for distilled water, tap water and $\mathrm{c}-\mathrm{c}$ in aqueous solution.

It can be assumed that the changes of $\Delta p$ and depth of liquid penetration into the capillary, besides the air flow rate, also are dependent on the liquid flow above the capillary generated by departed and moving bubbles. The liquid velocity above the capillary outlet is dependent on the departed bubble's diameter.

\section{NUMERICAL SIMULATIONS}

The air volume flow rate is one of the most important parameters that influence the maximum depth of liquid movement inside the glass capillary. However, the maximum depth of liquid penetration into the capillary can be indirectly modified by the liquid velocity above the capillary outlet and by modifications of the air pressure fluctuations in the gas supply system (Dzienis and Mosdorf, 2014; Mosdorf et al., 2017). The velocity of liquid flow above the capillary is modified by the diameter of the departed bubble. The schema of liquid flow around a single bubble above the capillary is shown in Fig. 5. 


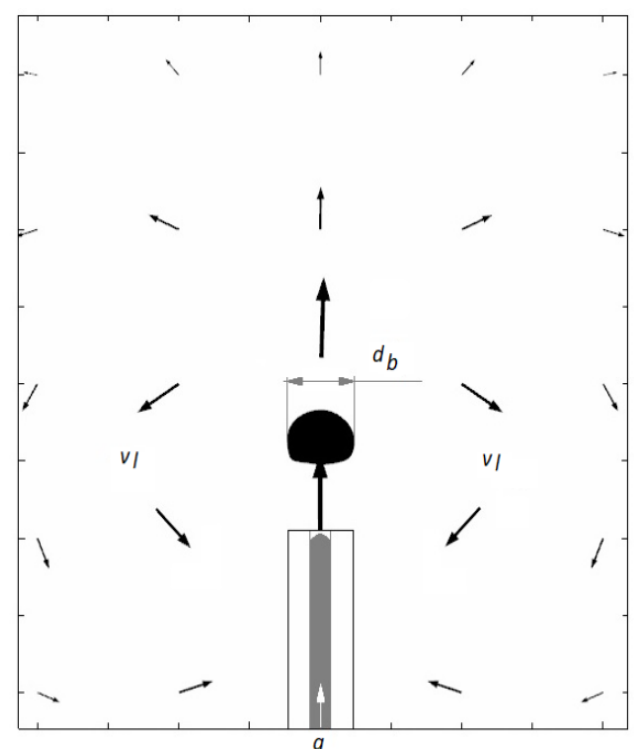

Fig. 5. Schema of liquid flow above the capillary after bubble departure.

The influence of bubble size on liquid velocity above the capillary outlet was numerically studied. The numerical model of bubble departure and bubble flow was prepared in COMSOL Multiphysics with the use of the CFD Module. In this model, the level set method was used (Osher and Sethian, 1988). In this method, the Navier-Stokes equation is solved using the following formulas:

$\nabla \cdot u=0$

$\rho \frac{\partial u}{\partial t}+\rho(u \cdot \nabla) u=\nabla \cdot\left[-p I+\mu\left(\nabla u+\nabla u^{T}\right)\right]+F+\rho g+$ $\sigma \kappa \delta n$

where: $g$ - gravity $\left(\mathrm{m} / \mathrm{s}^{2}\right), p$ - pressure $(\mathrm{Pa}), t$ - time $(\mathrm{s}), u$ - velocity $(\mathrm{m} / \mathrm{s})$ and I - identity matrix. The term $\sigma \kappa \delta n$ denotes the surface tension at the interface, where $\sigma$ - surface tension coefficient $(\mathrm{N} / \mathrm{m}), k$ - curvature, $n-$ is the unit normal to the interface and $\delta-$ Dirac delta function concentrated to the interface. The values of $k$, $n$ and $\delta$ are determined using the following expressions (Osher and Sethian, 1988):

$n=\frac{\nabla \phi}{|\nabla \phi|}$

$\kappa=\nabla\left(\frac{\nabla \phi}{|\nabla \phi|}\right)$

$\delta=6|\nabla \phi||\phi(1+\phi)|$

The level-set function is a smooth continuous function of $\phi$. Near the interface, the function $\phi$ changes smoothly from ' 0 ' to ' 1 '. Regions where $\phi<0.5$ show the occurrence of liquid and regions where $\phi>0.5$ show the occurrence of gas. The interface is represented by 0.5 contour of the function $\phi$ (Osher and Sethian, 1988).

The convection of the level set function is described by the following equation:

$\frac{\partial \phi}{\partial \mathrm{t}}+\mathrm{v} \cdot \nabla \phi=0$

The time evolution of the level-set function is described by the following equation (Osher and Sethian, 1988): $\frac{\partial \phi}{\partial t}+v \cdot \nabla \phi=\gamma \nabla \cdot\left(\varepsilon \nabla \phi-\phi(1-\phi) \frac{\nabla \phi}{|\nabla \phi|}\right)$

The parameter $\varepsilon$ determines the thickness of the region in which the function $\phi$ passes from ' 0 ' to ' 1 ' and is treated as the size of the elements of the mesh. The parameter $\gamma$ establishes the re-initialisation or stabilisation numbers of the level-set function (Kass et al., 1987).

The density $(\rho)$ and dynamic viscosity $(\mu)$ are described by the following formulas:

$\rho=\rho_{l}+\left(\rho_{a}-\rho_{l}\right) \phi$

$\mu=\mu_{l}+\left(\mu_{a}-\mu_{l}\right) \phi$

In Fig. 6, the liquid velocity above the capillary outlet, generated by the moving bubbles (with diameter equal to $3.8 \mathrm{~mm}, 4.0 \mathrm{~mm}$ and 4.2), is presented.

Increase of bubble diameter causes an increase of liquid flow above the capillary outlet. The velocity of liquid flow above the capillary outlet generated by smaller bubbles decreases slower in time than the velocity of liquid flow caused by bigger bubbles (Fig. 6).

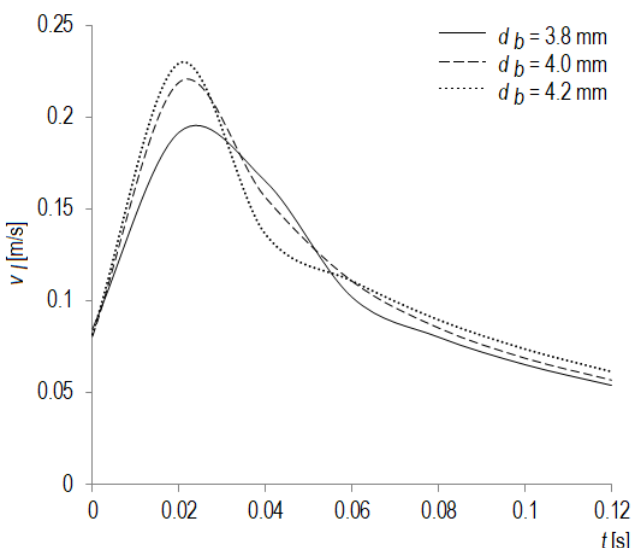

Fig. 6. The liquid velocity above the capillary outlet, generated by moving bubbles with diameter equal to: $3.8 \mathrm{~mm}, 4.0 \mathrm{~mm}$ and $4.2 \mathrm{~mm}$.

In order to determine the influence of liquid velocity above the capillary outlet on liquid penetration into the capillary, the numerical model in SciLab was prepared:

$\frac{d p_{c}}{d t}=\frac{p_{c}}{V_{c}}\left(q+\pi r_{n}^{2} \frac{d x_{l}}{d t}\right)$

$\frac{d}{d t}\left\{\left[0.5 \rho_{l} \pi r_{n}^{2} x_{l}+\rho_{l} \frac{4}{3} \pi\left(2 r_{n}\right)^{3}\right] \frac{d x_{l}}{d t}\right\}=F_{1}-F_{2}$

$F_{1}=-s \Delta p=-\pi r_{n}^{2}\left[p_{c}(t)-\left(p_{h}+\rho_{l} g\left(2 x_{l}\right)+2 \frac{\sigma}{r_{n}}-\right.\right.$

$\left.\left.\frac{\rho v_{p p}\left|v_{p p}\right|}{2}\right)\right]$

$F_{2}=2 x 8 \pi \mu_{l} x_{l} \frac{d x_{l}}{d t}$

where $r_{n}$ is the capillary radius $(m), x_{l}$ is the depth of the liquid penetration into the capillary $(\mathrm{m}), \rho_{l}$ is the liquid density $\left(\mathrm{kg} / \mathrm{m}^{3}\right), \mu_{\text {I }}$ is the liquid viscosity $(\mathrm{kg} / \mathrm{ms}), s$ is the cross-sectional area of the capillary $\left(\mathrm{m}^{2}\right), p_{c}$ is the gas pressure in the gas supply system $(\mathrm{Pa}), p_{h}$ is the hydrostatic pressure $(\mathrm{Pa}), v_{p p}$ is the velocity of the liquid around the growing bubble $(\mathrm{m} / \mathrm{s}), C_{0}=2$ drag coefficient of moving water inside the capillary.

This model include: the equation of motion of the liquid mass 
centre, i.e. Eq. (12), and the pressure changes in the air supply system, i.e. Eq. (11) (Ruzicka M.C. et al., 2009b; Dzienis P. and Mosdorf R. 2014). The force F1 in Eq. (13) is related to the pressure difference that occurs in the system. The force F2 in Eq. (14) is related to the resistance to the liquid movement in the capillary. The mass of the liquid that is involved in flooding the capillary is greater than the mass of the liquid inside the capillary. The mass of the liquid that is moving in the capillary is described by : $\frac{4}{3} \rho_{1} \pi r_{n}^{2} x_{l}+\rho_{1} \frac{4}{3} \pi\left(2 r_{n}\right)^{3}$. The reduced mass of the moving liquid is calculated assuming that the kinetic energy of the moving liquid is equal to the kinetic energy of the reduced mass. The profile of the velocity of liquid moving in the capillary is found to have a parabolic velocity profile. Consequently, the mass of the moving liquid is equal to to $\frac{4}{3} \rho_{1} \pi r_{n}^{2} x_{1}$, i.e. Eq. (14). The liquid movement stops when the centre of the liquid mass is equal to zero (Ruzicka M.C. et al., 2009b; Dzienis P. and Mosdorf R. 2014).
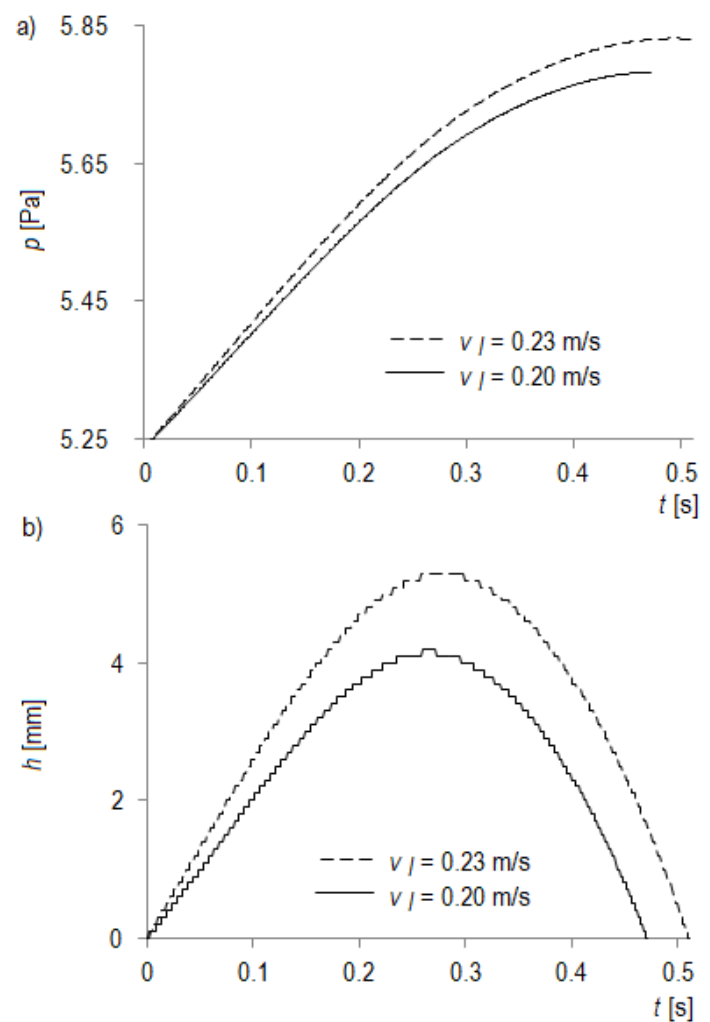

Fig. 7. The pressure changes and the liquid movement into the capillary for a single cycle of bubble departure for liquid velocity above the capillary outlet equal to $0.2 \mathrm{~m} / \mathrm{s}$ and $0.23 \mathrm{~m} / \mathrm{s}, q=0.020 \mathrm{l} / \mathrm{min}$, $\sigma=72 \mathrm{mN} / \mathrm{m}$ : (a) the pressure changes; and (b) the liquid movement into the capillary.

During the numerical simulations, the air volume flow rate was equal to $0.020 \mathrm{l} / \mathrm{min}$ and the surface tension was equal to $72.2 \mathrm{mN} / \mathrm{m}$. The results of the numerical analysis are shown in Fig. 7. In Fig. 7a, the pressure changes for a single cycle of bubble departure are shown, and in Fig. $7 \mathrm{~b}$, the liquid movement into capillary for a single cycle of bubble departure is presented.

The increase in liquid velocity above the capillary causes an increase in the bubble waiting time (Fig. 7a and b). Moreover, the changes in liquid velocity above the capillary outlet cause changes in pressure. When the velocity becomes lower, then the value of $p_{\max }$ decreases. Consequently, the value of $\Delta p$ became lower too (Fig. 7a). When the liquid velocity decreases, the depth of the liquid's penetration inside the capillary increases (Fig. 7b).

The experimental and numerical investigations show that the process of liquid penetration into the capillary during bubble departures is very complex and depends on many factors. The air volume flow rate and the surface tension have the greatest impact on the changes of pressure $(\Delta p)$ during a single cycle of bubble departure, which determine the depth of liquid penetration into the capillary. Moreover, the values of $\Delta p$ and the depth of liquid penetration can be modified by variations in liquid velocity above the capillary outlet.

\section{CONCLUSIONS}

In the present paper, the influence of the size of departed bubbles on the liquid velocity above the capillary and on the depth of liquid penetration into the capillary was experimentally and numerically studied.

The experimental results show that the bubble diameter is dependent on the surface tension and the flow rate of the air volume supplied to the capillary. An increase in surface tension causes an increase in the departing bubble's diameter. Moreover, the changes of surface tension influence the changes of pressure $(\Delta p)$ during a single cycle of bubble departure. Further, an increase in surface tension causes an increase in $\Delta p$. These changes are responsible for the depth of liquid penetration into the capillary. Consequently, an increase in surface tension causes an increase in the depth of liquid penetration.

Based on the results of the numerical investigations, we can see that the liquid velocity above the capillary can modify the changes of pressure $(\Delta p)$ during a single cycle of bubble departure. When the liquid velocity decreases, $\Delta p$ decreases. When $\Delta p$ decreases, the depth of liquid penetration into the capillary decreases, too.

This effect is visible for the case in which bubbles were generated in distilled water. It can be assumed that, on the one hand, the increase in air volume flow rate causes the decrease in $\Delta p$. On the other hand, a very large increase in bubble diameter $v s$. increase in air volume flow rate causes the increase in $\Delta p$. Consequently, $\Delta p$ increases slightly (with increase in air volume flow rate) and the liquid penetration into the capillary is almost constant for changes of air volume flow rate.

In order to confirm the numerical investigation of the influence of liquid velocity above the capillary on liquid penetration into the capillary, experimental investigations using the particle image velocimetry method will be performed.

\section{REFERENCES}

1. Aoyama, S., Hayashi, K. Hosokawa, S., Tomiyama A., (2016), Shapes of ellipsoidal bubbles in infinite stagnant liquids Int. J. Multiphase. Flow, 79, 23-30.

2. Augustyniak, J., Perkowski, D. M., (2021), Compound analysis of gas bubble trajectories with help of multifractal algorithm, Exp Thermal Fluid Sci., 124, 110351.

3. Cano-Lozano, J.C., Bolaños-Jiménez, R., Gutiérrez-Montes, C., Martínez-Bazán, C., (2017), On the bubble formation under mixed injection conditions from a vertical needle. Int J Multiphase FLows. 97, 23-32. 
4. Cieslinski, J.T., Mosdorf, R., (2005), Gas bubble dynamics experiment and fractal analysis, Int. J. Heat Mass Transfer 48 (9) 18081818.

5. Dukhin S.S., Koval'chuk V.I., Fainerman V.B., Miller R.,(1998b), Hydrodynamic processes in dynamic bubble pressure experiments Part 3. Oscillatory and aperiodic modes of pressure variation in the capillary, Colloids and Surfaces A: Physicochemical and Engineering Aspects 141, s 253-267.

6. Dukhin S.S., Mishchuk N.A., Fainerman V.B., Miller R., (1998a)., Hydrodynamic processes in dynamic bubble pressure experiments 2 . Slow meniscus oscillations, Colloids and Surfaces A: Physicochemical and Engineering Aspects 138 s. 51-63.

7. Dzienis, P., Mosdorf, R., (2013), Synchronization of data recorded using acquisition stations with data from camera during the bubble departure. Adv. Sci. Technol. Res. J. 7 no 20, 29-34.

8. Dzienis, P., Mosdorf, R., (2014) Stability of periodic bubble departures at a low frequency. Chemical Engineering Science. 109, 171182.

9. Farhat, M., Chinaud, M., Nerisson, P., Vauquelin, P., (2021), Characterization of bubbles dynamics in aperiodic formation, Int $\mathrm{J}$ Heat Mass Transfer, 180, 121646.

10. Kass, M., Witkin, A., Terzopoulos, D., (1987), Snakes - Active Contour Models., Int. J. Comp. Vis., 1, 4, 321-331.

11. Koval'chuk V.I., Dukhin S.S., Fainerman V.B., Miller R., (1999), Hydrodynamic processes in dynamic bubble pressure experiments. 4. Calculation of magnitude and time of liquid penetration into capillaries, Colloids and Surfaces A: Physicochemical and Engineering Aspects 151, s 525-536.

12. Leifer, I., Tang, D., (2007), The acoustic signature of marine seep bubbles. J. Acoust. Soc. Am. 121, 35-40.

13. Liu, L., Yan, H., Zhao, G., (2015), Experimental studies on the shape and motion of air bubbles in viscous liquids Exp. Therm. Fluid Sci., 62, 109-121.
14. Mosdorf, R., Shoji, M.,(2003), Chaos in bubbling - nonlinear analysis and modelling, Chem. Eng. Sci. 58 (2003) 3837-3846.

15. Osher, S., Sethian, J. A., (1988), Fronts Propagating with Curvature-Dependent Speed: Algorithms Based on Hamilton-Jacobi Formulations. J. Comp. Phys., 79, 1, pp. 12-49.

16. Ruzicka M.C., R. Bunganic R., Draho`s J., (2009b), Meniscus dynamics in bubble formation. Part II: Model, Chem. Eng. Res. Des., 87, s. 1357-1365.

17. Ruzicka, M.C., Bunganic, R. Drahos, J., (2009a), Meniscus dynamics in bubble formation. Part I: Experiment. Chem. Eng. Res. Des. 87: 1349-1356.

18. Stanovsky P., Ruzicka M.C., Martins A., Teixeira J.A, (2011) Meniscus dynamics in bubble formation: A parametric study, Chemical Engineering Science, 66, s. 3258-3267.

19. Vázquez, A., Manasseh, R., Chicharro, R. (2015), Can acoustic emissions be used to size bubbles seeping from a sediment bed. 131, 187-196.

20. Zang L., Shoji M., (2001), Aperiodic bubble formation from a submerged orifice, Chemical Engineering Science 56, 5371-5381.

Acknowledgment: I gratefully acknowledge the help of Romuald Pawe Mosdorf (Professor), from Faculty of Mechanical Engineering, Bialystok University of Technology in creating the model of liquid penetration into the capillary.

Paweł Dzienis: (iD https://orcid.org/0000-0001-9200-8760 